\title{
Genome-wide transcriptomic and phylogenetic analyses reveal distinct aluminum-tolerance mechanisms in the aluminum-accumulating species buckwheat (Fagopyrum tataricum)
}

\author{
Haifeng Zhu ${ }^{1 \dagger}$, Hua Wang ${ }^{2+}$, Yifang Zhu' ${ }^{1}$, Jianwen Zou', Fang-Jie Zhao ${ }^{1}$ and Chao-Feng Huang ${ }^{1 *}$
}

\begin{abstract}
Background: Similar to common buckwheat (Fagopyrum esculentum), tartary buckwheat (Fagopyrum tataricum) shows a high level of aluminum (Al) tolerance and accumulation. However, the molecular mechanisms for Al detoxification and accumulation are still poorly understood. To begin to elucidate the molecular basis of Al tolerance and accumulation, we used the Illumina high-throughput mRNA sequencing (RNA-seq) technology to conduct a genome-wide transcriptome analysis on both tip and basal segments of the roots exposed to Al.

Results: By using the Trinity method for the de novo assembly and cap3 software to reduce the redundancy and chimeras of the transcripts, we constructed 39,815 transcripts with an average length of 1184 bp, among which 20,605 transcripts were annotated by BLAST searches in the NCBI non-redundant protein database. Gene Ontology (GO) and Kyoto Encyclopedia of Genes and Genomes (KEGG) enrichment analysis showed that expression of genes involved in the defense of cell wall toxicity and oxidative stress was preferentially induced by Al stress. Our RNA-seq data also revealed that organic acid metabolism was unlikely to be a rate-limiting step for the Al-induced secretion of organic acids in buckwheat. We identified two citrate transporter genes that were highly induced by Al and potentially involved in the release of citrate into the xylem. In addition, three of four conserved Al-tolerance genes were found to be duplicated in tartary buckwheat and display diverse expression patterns.
\end{abstract}

Conclusions: Nearly 40,000 high quality transcript contigs were de novo assembled for tartary buckwheat, providing a reference platform for future research work in this plant species. Our differential expression and phylogenetic analysis revealed novel aspects of Al-tolerant mechanisms in buckwheat.

Keywords: Aluminum tolerance, Al-tolerance genes, Buckwheat, Homolog, Organic acid, Transcriptome

\section{Background}

Aluminum (Al) toxicity is a major limiting factor for crop production on acid soils, which make up over $30 \%$ of the world's arable soils and up to $70 \%$ of the potential arable land [1]. On acidic soils with $\mathrm{pH}$ below 5.5, phytotoxic forms of $\mathrm{Al}$ (mainly $\mathrm{Al}^{3+}$ ) are solubilized into the soil solution, which inhibit root growth and thereafter limit water and mineral nutrient uptake, resulting

\footnotetext{
* Correspondence: chaofeng.huang@njau.edu.cn

${ }^{\dagger}$ Equal contributors

'State Key Laboratory of Crop Genetics and Germplasm Enhancement, College of Resources and Environmental Science, Nanjing Agricultural

University, Nanjing 210095, China

Full list of author information is available at the end of the article
}

in losses of crop yield [2]. To grow on Al-toxic environments, some plant species have evolved resistance mechanisms that enable them to tolerate toxic levels of Al.

$\mathrm{Al}$-activated organic acid release from roots is a well-documented mechanism of $\mathrm{Al}$ detoxification [3,4]. Organic acids such as malate, citrate and oxalate are able to chelate $\mathrm{Al}$ and thereby attenuate $\mathrm{Al}$ toxicity. Different plants secrete different organic acids to detoxify Al. For example, wheat (Triticum aestivum), oilseed rape (Brassica napus) and Arabidopsis thaliana secrete malate after exposure to $\mathrm{Al}$ stress [5-7], while Al-tolerant cultivars of snapbean (Phaseolus vulgaris), rice bean (Vigna umbellata), maize (Zea mays), and soybean (Glycine max) 
release citrate in response to $\mathrm{Al}$ stress [8-12]. Oxalate can be secreted from the roots of buckwheat, tomato and spinach (Spinacia oleracea) upon exposure to $\mathrm{Al}$ stress [13-16]. Recently, genes responsible for the $\mathrm{Al}$-activated secretion of malate and citrate have been identified. Sasaki et al. [17] cloned the first Al-resistant gene ALMT1 in wheat, which encodes a plasma membrane transporter to transport malate from root cells to the rhizosphere for the chelation and detoxification of Al. Genes for citrate secretion were independently identified in barley [18] and sorghum [19], which were found to encode members of the multidrug and toxic compound extrusion (MATE) family. To date, genes involved in oxalate release have not been identified.

Using mutant screening and map-based gene cloning approaches on the model plants, rice and Arabidopsis, recent studies have unraveled some common Al-tolerant mechanisms in plants. ART1/STOP1 is a C2H2-type zincfinger transcription factor, which is required for $\mathrm{Al}$ tolerance through regulation of downstream Al tolerance genes in both rice and Arabidopsis [20,21]. STAR1 and STAR2/ALS3 encode a nucleotide-binding domain and a transmembrane domain of an ABC (ATP-binding cassette) transporter, respectively. STAR1 and STAR2/ASL3 form a complex to transport UDP-glucose for the modification of cell walls thereby detoxifying $\mathrm{Al}$ [22-24]. ALS1 encodes a half-size $A B C$ transporter and is involved in sequestering $\mathrm{Al}$ into the vacuoles for the internal detoxification of $\mathrm{Al}$ $[25,26]$. Although the functions of STAR1, STAR2/ALS3 and ALS1 in Al tolerance are conserved in plants, their expression patterns differ between rice and Arabidopsis. In general, the expression level and the level of induction by $\mathrm{Al}$ stress of these genes are higher in the Al-tolerant species rice than in the Al-sensitive species Arabidopsis, suggesting that $\mathrm{Al}$-tolerant species may require increased expression of these conserved Al-tolerance genes to overcome Al stress.

Common buckwheat (Fagopyrum esculentum) is an Al-tolerant species and can accumulate $\mathrm{Al}$ to levels as high as 15,000 ppm in leaves, when grown on acid soils, without displaying symptoms of Al toxicity [27]. Physiological studies have demonstrated that common buckwheat secretes oxalate to detoxify $\mathrm{Al}$ externally and utilizes oxalate to chelate and sequester $\mathrm{Al}$ in the vacuoles of both roots and shoots for internal detoxification $[13,14,28]$. Although oxalate is required for Al translocation in buckwheat, $\mathrm{Al}$ in the xylem appears to be complexed with citrate instead of oxalate, suggesting that $\mathrm{Al}$ may undergo a ligand exchange from oxalate to citrate when $\mathrm{Al}$ is transported into the xylem [29]. However, understanding the molecular mechanisms of $\mathrm{Al}$ tolerance in buckwheat has been hampered by the lack of the genomic sequence and transcriptomic data under $\mathrm{Al}$ stress.
Recent advances in high-throughput mRNA sequencing (RNA-seq) offer the capability to discover new genes and transcripts and to quantify gene expression simultaneously. In the present study, we used the RNA-seq technique to analyze the transcriptome of different root zones of tartary buckwheat (Fagopyrum tataricum) in response to $\mathrm{Al}$ treatment. Tartary buckwheat was chosen in our study because it is an Al-accumulating species [30] but unlike common buckwheat, is self-pollinating, which makes it easier to assemble transcripts and to conduct further gene function analysis. We constructed high-quality genome-wide transcripts and examined the expression profile of Al-responsive genes in this buckwheat species. Combined with quantitative RT-PCR and phylogenetic analysis, our results revealed novel aspects of Al-tolerant mechanisms in tartary buckwheat.

\section{Results}

Al accumulation pattern in tartary buckwheat

To compare $\mathrm{Al}$ accumulation by tartary buckwheat and common buckwheat, we exposed plants of both species to different $\mathrm{Al}$ concentrations for $8 \mathrm{~d}$ intermittently in a hydroponic experiment. Both species accumulated appreciable amounts of $\mathrm{Al}$ in the roots and shoots in the control treatment (Figure 1A and B), suggesting that both buckwheat species efficiently took up the background level of $\mathrm{Al}$ in the nutrient solution. In the treatments with $10-50 \mu \mathrm{M} \mathrm{Al}$, tartary buckwheat accumulated significantly more $\mathrm{Al}$ in the roots than common buckwheat (Figure 1A). Tartary buckwheat accumulated higher concentrations of $\mathrm{Al}$ in the shoots than common buckwheat in the $10 \mu \mathrm{M}$ $\mathrm{Al}$ treatment, whereas shoot $\mathrm{Al}$ concentrations were similar between the two species in the higher $\mathrm{Al}$ treatments $(20$ and $50 \mu \mathrm{M})$ (Figure 1B).

In both species, the $\mathrm{Al}$ translocation efficiency from roots to shoots decreased with increasing $\mathrm{Al}$ concentration in the solution (Figure 1C). The shoot to root $\mathrm{Al}$ concentration ratio in tartary buckwheat decreased from 0.52 in the control treatment to 0.08 in the $50 \mu \mathrm{M} \mathrm{Al}$ treatment. This result suggests that xylem loading of $\mathrm{Al}$ might be the rate-limiting step controlling $\mathrm{Al}$ accumulation in the shoots in buckwheat.

\section{De novo assembly of the transcripts and annotation}

For RNA-seq analysis, tartary buckwheat plants were treated with $50 \mu \mathrm{M} \mathrm{Al}$ for a short period of time $(6 \mathrm{~h})$. At this concentration root elongation was inhibited by $76 \%$ compared to the control (data not shown). Root tips and basal roots from both the control and + Al treatments were sampled for RNA isolation and Illumina paired-end RNA-seq. RNA-seq generated a range of $35.1 \sim 46.6$ million clean reads on each sample (Additional file 1: Table S1). In total there were 267.4 million clean reads from all samples with an average length of 100 nucleotides per read 
A

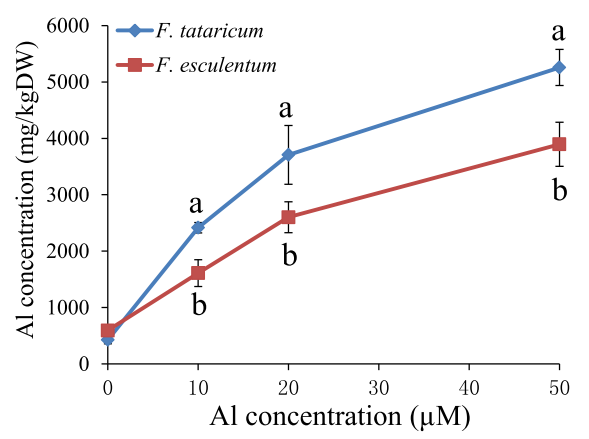

C

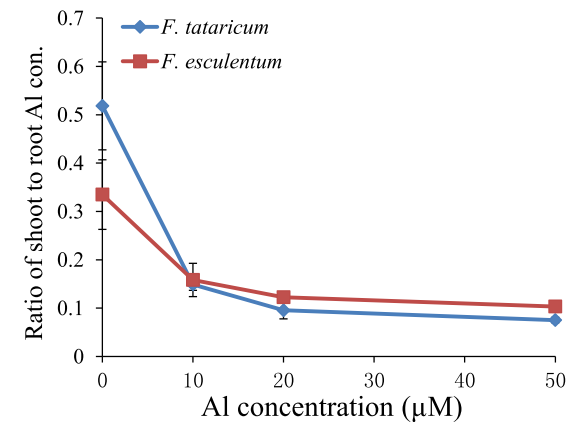

B

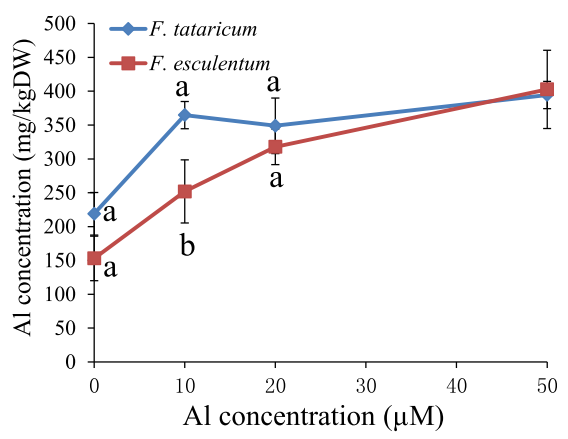

Figure $1 \mathrm{Al}$ accumulation in roots and shoots of Fagopyrum tataricum and Fagopyrum esculentum. Two-week-old seedlings were exposed to a series of $\mathrm{Al}$ concentrations $(0,10,20,50 \mu \mathrm{M} \mathrm{Al})$ for $8 \mathrm{~d}$ intermittently. The Al concentrations in roots $(\mathbf{A})$ and shoots (B) and the ratio of shoot to root Al concentrations $(\mathbf{C})$ were analyzed, respectively. Data are means $\pm S D(n=4)$. Means with different letters are significantly different $(P<0.05$, Tukey test).

and a GC content of $47.84 \%$ GC. These were used for the assembly of the transcripts. Because the genome sequence of buckwheat is not available, a de novo assembly method, the Trinity method [31,32], was used to construct the transcripts and 58,438 transcript contigs were assembled. In order to reduce the redundancy and chimeras of the transcripts, we used cap3 software to combine highly similar transcripts and retain the longest transcripts with the highest read coverage, and removed the transcripts with the lowest read coverage [31]. As a result, the number of contigs was further reduced to 39,815 (Additional file 2: Table S2). The assembled contigs had a length distribution from 201 to $25,284 \mathrm{bp}$, with an average length of $1184 \mathrm{bp}$ (Figure 2). The average coverage for each assembled contig is 508 reads per base, indicating a high read coverage of the contigs.

Recently, Logacheva et al. [33] performed transcriptome sequencing in F. tataricum by 454 sequencing. In comparison with their results, we produced more assembled contigs $(39,815$ vs 25,401$)$ and a longer average contig length (1184 vs 703) (Table 1). Moreover, $89.4 \%$ of the contigs from the study of Logacheva et al. [33] were covered in our assembled transcripts. Therefore, the assembled contigs in our study should provide a useful resource for future research on F. tataricum.
BLAST searches revealed that 20,605 of 39,815 contigs had significant matches in the NCBI non-redundant protein database. Gene Ontology (GO) analysis of the matched contigs identified 8110 genes that were categorized into different GO groups (Figure 3). Some of the gene categories are partially redundant, which led to some genes being categorized into more than one group. In the molecular function category, genes assigned to the "catalytic activity" and "binding (other binding)" groups are highly enriched. In the cellular component category, genes in the "cell" and "intracellular" groups were the most abundant. In the biological process category, the "cellular process" and "macromolecule metabolism" groups contain the highest number of genes (Figure 3).

\section{Calculation and validation of RNA-seq expression data}

The expression of each gene from the RNA-seq data was calculated by reads per kilobase of exons per million mapped reads. Although we used all 6 samples for the assembly of the transcripts, all genes identified had read coverage on each sample (data not shown), suggesting that our RNA sequencing of each sample was deep enough to allow expression analyses for all the genes. To verify the RNA-seq expression data, we selected 14 genes displaying diverse expression profiles in the root 


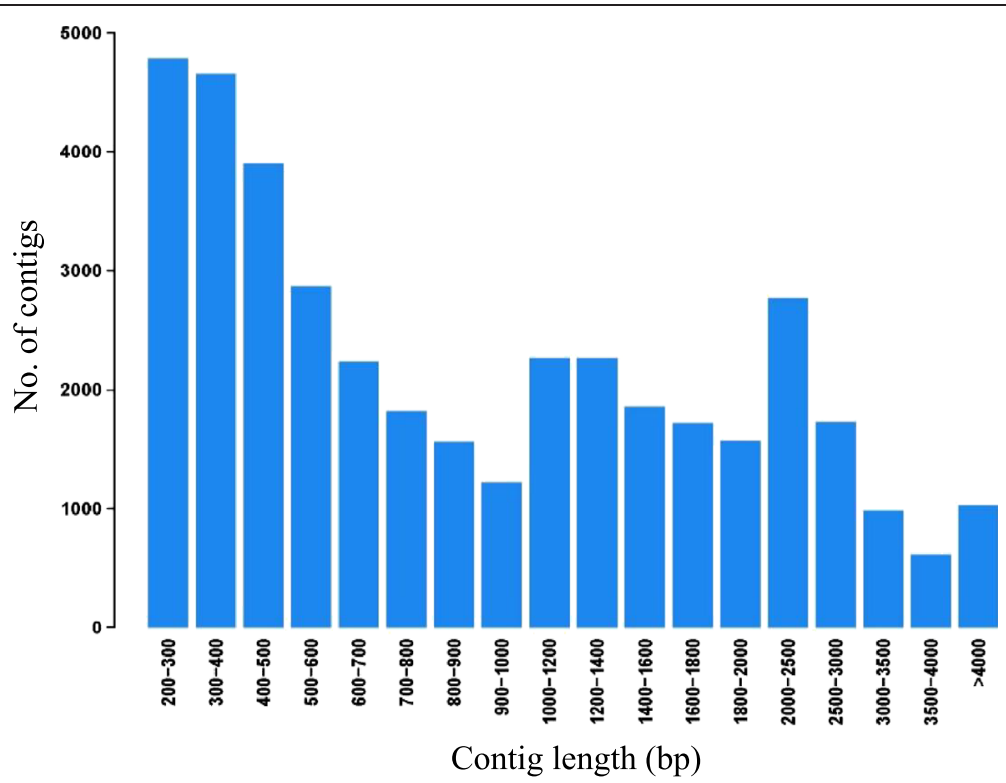

Figure 2 Distribution of the length of transcript assembly contigs.

tips and/or basal roots for real-time RT-PCR analysis. A significant correlation $\left(R^{2}=0.89\right)$ was observed between two data sets (Figure 4). These results confirm the reliability of our RNA-seq expression data.

\section{Global effect of Al stress on gene expression}

In the root tips, there are 1487 genes up-regulated and 775 genes down-regulated ( $\mid \log _{2} \mathrm{FC}$ (fold change) $\mid \geq 1$, FDR (false discovery rate) $\leq 0.001$ ) under $\mathrm{Al}$ stress (Additional file 3: Table S3). Although root tips are known to be the main sites for $\mathrm{Al}$ detoxification, we found that there were also a large number of genes affected in the basal roots by $\mathrm{Al}$ stress, with 1719 genes being upregulated and 1287 genes being downregulated (Additional file 4: Table S4). GO enrichment analysis showed that the upregulated genes in both root tips and basal roots were significantly overrepresented in four categories: "Response to stimulus", "Antioxidant activity", "Extracellular" and "Cell death" (Table 2) (FDR $\leq 0.001)$, although the upregulated genes in

Table 1 Comparison of Illumina sequencing data with reported 454 sequencing data

\begin{tabular}{lll}
\hline & Illumina sequencing & 454 sequencing \\
\hline No. of reads & $267,438,632$ & 229,031 \\
Average length of reads & 100 & 341 \\
Total nucleotides & 26.7 billion & 0.078 billion \\
No. of assembled contigs & 39,815 & 25,401 \\
$\begin{array}{l}\text { Average length of contigs } \\
\text { (Min-max) }\end{array}$ & $1184(201-25284)$ & $703(46-3298)$ \\
No. of reads per contig, mean & $3008(100)$ & $7.5(2-295)$ \\
(min-max) & & \\
\hline
\end{tabular}

the "Cell death" group were not significantly enriched in the basal roots due to the strict cut-off criteria used. This result suggests that defensive genes and genes encoding extracellular-localized proteins, such as cell wall components, were preferentially induced in expression by $\mathrm{Al}$ stress. The upregulated genes in the root tips and basal roots were also subjected to KEGG pathway enrichment analysis. Genes in two pathways, "Xenobiotics biodegradation and metabolism" and "Lipid metabolism", were significantly enriched in both the root tip and basal root region (Table 3). The enrichment of genes in the lipid metabolism pathway supports the observation that $\mathrm{Al}$ can interfere with the function of the plasma membrane and induce its lipid peroxidation [34,35]. Together, these results suggest that $\mathrm{Al}$ toxicity can act on both the root tip and the basal root region and that both regions have evolved some common mechanisms of $\mathrm{Al}$ responsiveness in buckwheat. Further support for this statement came from the fact that a large portion of the upregulated or downregulated genes were shared between the root tips and the basal root region, with 946 and 369 genes being upregulated and downregulated in both root regions, respectively (Figure 5). By contrast, genes in "Carbohydrate metabolism" pathway were only significantly enriched in the root tip region, and genes in four pathways, "signal transduction", "Environmental adaptation", "Immune system" and "Sensory system" were overrepresented in the basal roots but not in the root tips under $\mathrm{Al}$ stress (Table 3). Expression analysis also showed that some genes were upregulated or downregulated only in the root tips or the basal roots (Figure 5; Additional file 3: Table S3, Additional file 4: Table S4). Therefore, the root tip and 


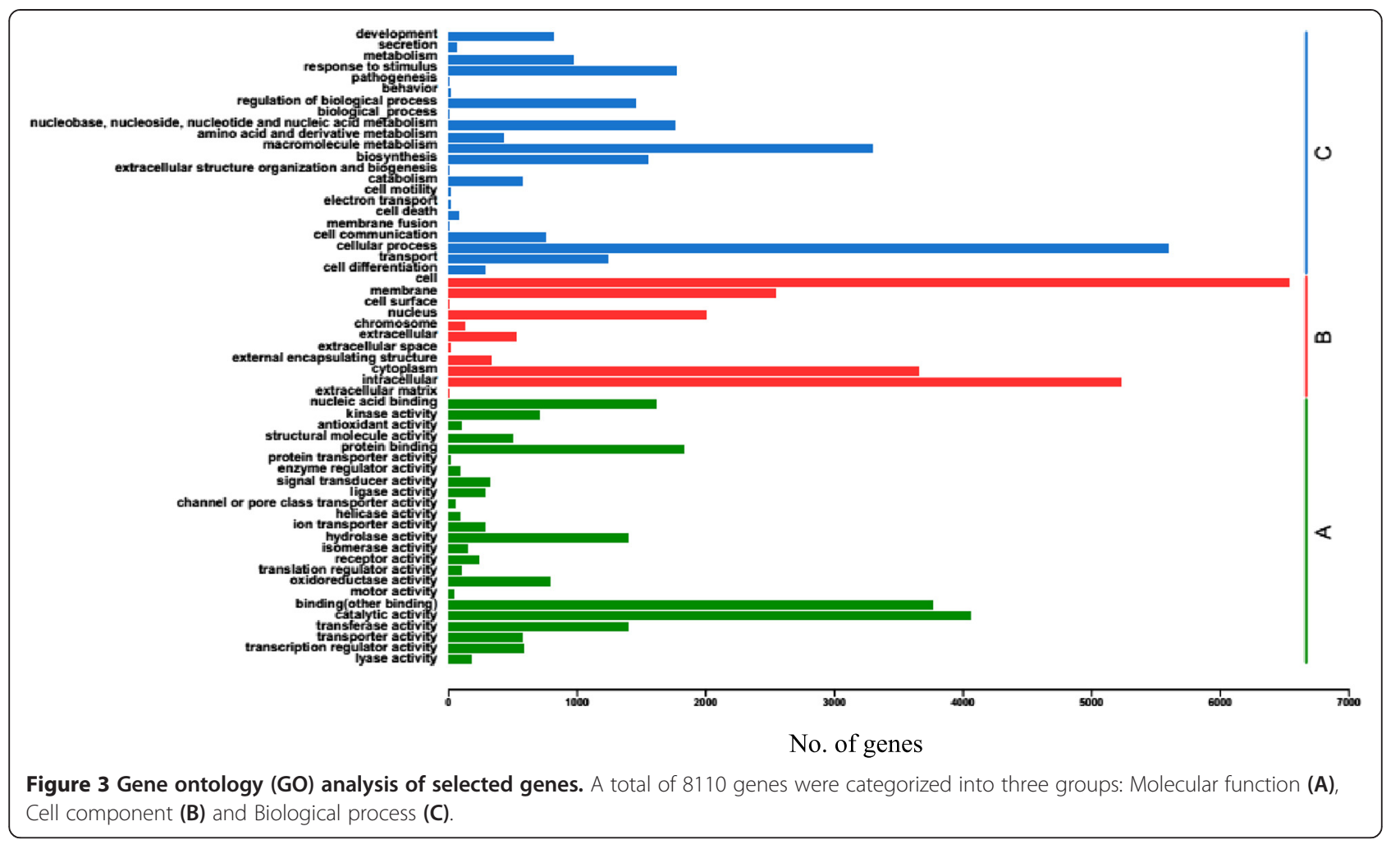

basal root region may also possess different mechanisms of $\mathrm{Al}$ responsiveness in buckwheat.

Effect of Al on the expression of organic acid metabolism and secretion-related genes

Secretion of oxalate from the root tips in response to $\mathrm{Al}$ and chelation of $\mathrm{Al}$ by oxalate within root cells are well-

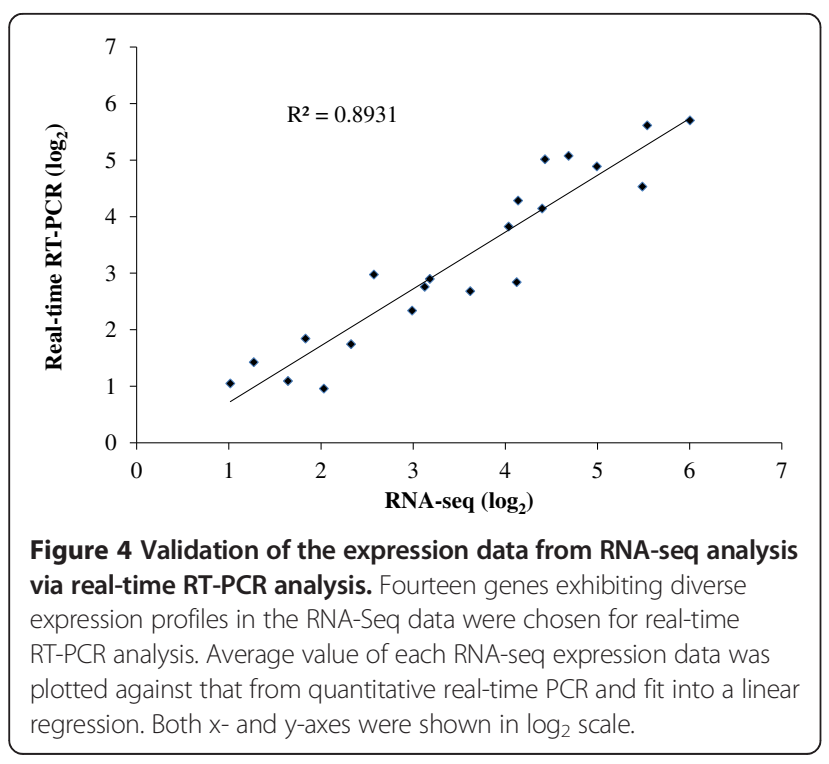

characterized mechanisms of $\mathrm{Al}$ tolerance in buckwheat $[14,28,36,37]$. We therefore investigated the effect of $\mathrm{Al}$ on the expression of genes involved in organic acid synthesis or metabolism. The results showed that the expression of genes putatively involved in the tricarboxylic acid cycle, including key enzymes such as malate dehydrogenase and citrate synthase, was not induced by $\mathrm{Al}$ stress (Additional file 5: Figure S1), which is consistent with evidence that organic acid metabolism is not a ratelimiting step for $\mathrm{Al}$-induced release of organic acids [38-40]. Interestingly, we found that two genes belonging to the MATE (Multidrug And Toxic compound Extrusion) family were induced in expression in both the root tips and basal roots by Al stress (Figure 6). Phylogenetic analysis indicated that the two MATE members, FtFRDL1 and FtFRDL2, clustered with the citrate transporter AtFRD3, a founding member of the FRD3 subfamily (Figure 6A). Although the basal expression of FtFRDL1 in the absence of $\mathrm{Al}$ was higher than that of FtFRDL2, the latter was more induced by $\mathrm{Al}$, resulting in a similar expression level of the two genes after exposure to the $\mathrm{Al}$ stress (Figure 6B). The MATE genes from the FRD3 clade have been shown to be involved in transporting citrate $[18,19,41,42]$. Although $\mathrm{Al}$-activated citrate secretion is not the Al-tolerance mechanism in buckwheat, citrate might be transported into the xylem for Al chelation and translocation [29]. Therefore, it is 
Table 2 Gene ontology enrichment analysis of upregulated genes in root tips and basal roots exposed to Al stress

\begin{tabular}{|c|c|c|c|c|c|}
\hline & \multirow{2}{*}{$\begin{array}{l}\text { No. of genes } \\
\text { in the whole } \\
\text { transcriptome }\end{array}$} & \multicolumn{2}{|l|}{ Root tip region } & \multicolumn{2}{|l|}{ Basal root region } \\
\hline & & No. of upregulated genes & FDR & No. of upregulated genes & FDR \\
\hline Response to stimulus & $1772(8110)$ & $165(484)$ & $3.20 \mathrm{E}-09$ & $193(526)$ & 1.67E-14 \\
\hline Antioxidant activity & $98(8110)$ & $19(484)$ & 2.77E-05 & $67(526)$ & 5.96E-07 \\
\hline Extracellular & $528(8110)$ & $57(484)$ & $6.03 \mathrm{E}-05$ & $18(526)$ & 2.57E-04 \\
\hline Cell death & 74 (8110) & 14 (484) & 3.45E-04 & $13(526)$ & $3.42 \mathrm{E}-03$ \\
\hline
\end{tabular}

possible that the two MATE genes are involved in the release of citrate into the xylem.

\section{Expression and phylogenetic analysis of Al-tolerance gene homologs}

A number of genes required for $\mathrm{Al}$ tolerance in rice and Arabidopsis have been cloned and characterized recently. To understand the mechanisms of $\mathrm{Al}$ tolerance in the $\mathrm{Al}$ hyperaccumulator buckwheat, we performed expression and phylogenetic analysis of homologs of four conserved Al-tolerance genes in rice and Arabidopsis, ART1/STOP1, ALS1, STAR1 and STAR2/ALS3. We identified two homologs of ART1, namely ARL1 and ARL2 (ART1-Like) in buckwheat. Phylogenetic analysis indicated that both ARL1 and ARL2 are closer to Arabidopsis STOP1 than to rice ART1 (Figure 7A), suggesting that the duplication event of ART1 in buckwheat happened after the dicotmonocot split. Real-time RT-PCR analysis showed that both $A R L 1$ and $A R L 2$ were equally expressed in the root tips and basal roots, and their expression was not affected by the $\mathrm{Al}$ treatment (Figure $8 \mathrm{~A}$ ).

There were two ALS1 homologs found in buckwheat. Interestingly, one of the ALS1 homologs (FtALOL1, ALS One-Like 1) is closer to rice OsALS1, whereas the other (FtALOL2) is closer to Arabidopsis AtALS1 (Figure 7B), suggesting that ALS1 duplication in buckwheat occurred before the split of monocots and dicots. Expression analysis showed that FtALOL2 transcript accumulation was higher than that of FtALOL1 in the roots, and that FtALOL2 expression was induced by the $\mathrm{Al}$ stress, to a greater extent in the root tips compared with the basal roots (Figure $8 \mathrm{~B}$ ).
By contrast, the expression of FtALOL1 was downregulated by the $\mathrm{Al}$ treatment.

We identified two homologs of STAR1, STOL1 and STOL2 (STAR-One Like), in buckwheat. Both STOL1 and STOL2 fall into the dicot group (Figure 7C), suggesting that STAR1 was duplicated in buckwheat after the evolutionary divergence of dicots and monocots. Quantitative RT-PCR analysis showed that the expression level of STOL2 was more than 50 fold higher than that of STOL1 in the roots (Figure $8 \mathrm{C}$ ), and the expression of STOL2 was induced in both the root tips and the basal root region by $\mathrm{Al}$ stress, but that of STOL1 was not. These results suggest that STOL2 may play a major role for $\mathrm{Al}$ tolerance in buckwheat roots. Whether STOL1 plays an important role in the shoots requires further investigation. In contrast to STAR1, there was only one homolog of STAR2 in buckwheat (Figure 7D). The expression of FtSTAR2 in both the root tips and the basal root region was highly induced by $\mathrm{Al}$ stress (Figure 8D).

\section{Discussion}

Similar to common buckwheat, tartary buckwheat was able to accumulate high levels of $\mathrm{Al}$ in the roots and shoots in a short-term hydroponic experiment (Figure 1). This result is consistent with a recent report showing that tartary buckwheat shares similar mechanisms of $\mathrm{Al}$ detoxification and accumulation with common buckwheat species [30].

Through Illumina high-throughput mRNA sequencing and de novo assembly of the transcripts with an optimized method, we constructed nearly 40,000 transcript

Table 3 KEGG enrichment analysis of upregulated genes in root tips and basal roots exposed to Al stress

\begin{tabular}{|c|c|c|c|c|c|}
\hline \multirow[t]{2}{*}{ KEGG pathway } & \multirow{2}{*}{$\begin{array}{l}\text { No. of genes } \\
\text { in the whole } \\
\text { transcriptome }\end{array}$} & \multicolumn{2}{|l|}{ Root tip region } & \multicolumn{2}{|l|}{ Basal root region } \\
\hline & & No. of upregulated genes & FDR & No. of upregulated genes & FDR \\
\hline Xenobiotics biodegradation and metabolism & $188(3282)$ & $28(162)$ & 4.67E-07 & $28(180)$ & $1.34 \mathrm{E}-06$ \\
\hline Lipid metabolism & 307 (3282) & $30(162)$ & 7.19E-04 & $31(180)$ & 7.81E-04 \\
\hline Carbohydrate metabolism & $710(3282)$ & $55(162)$ & 7.19E-04 & $44(180)$ & 2.33E-01 \\
\hline Signal transduction & $669(3282)$ & $45(162)$ & $3.02 \mathrm{E}-02$ & $65(180)$ & $1.34 \mathrm{E}-06$ \\
\hline Environmental adaptation & $144(3282)$ & $15(162)$ & $1.26 \mathrm{E}-02$ & $22(180)$ & 1.25E-05 \\
\hline Immune system & $251(3282)$ & $10(162)$ & $9.54 \mathrm{E}-01$ & $31(180)$ & $1.64 \mathrm{E}-05$ \\
\hline Sensory system & $23(3282)$ & $2(162)$ & 2.25E-01 & $6(180)$ & $6.82 \mathrm{E}-04$ \\
\hline
\end{tabular}


A

Up-regulated genes

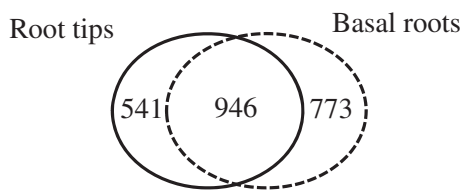

B

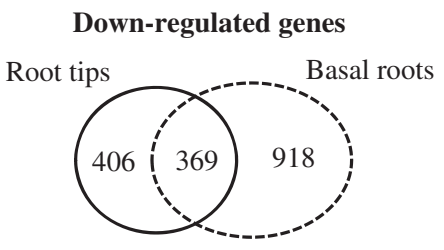

Figure 5 Genes upregulated and downregulated in the root tips and basal roots after exposure to Al stress. (A) Diagrams showing the genes upregulated by Al in the root tips (black circle) and basal roots (dotted circle). (B) Diagrams showing the genes downregulated by Al in the root tips and basal roots.

contigs of high quality in tartary buckwheat (F. tataricum). Compared with previous 454 sequencing in F. tataricum [33], our high-throughput mRNA sequencing generated 300 fold more nucleotides and therefore enabled us to assemble more contigs and obtain longer transcripts (Table 1). When we cloned full transcripts of the gene homologs of FRD3, ART1, ALS1, STAR1 and STAR2 by 5 '-RACE and 3'-RACE PCR in buckwheat, we found that in fact all the homologs had full length open reading frames (ORFs) in our assembled contigs, whereas the ORFs of the homologs from previous 454 sequencing data were incomplete (Data not shown). Thus, our assembled transcripts provide a platform for future research on buckwheat.

Our differential expression analysis of RNA-seq data revealed that a large number of genes upregulated or downregulated by Al stress were shared in the root tips and basal roots (Figure 5), which suggested that at the cellular level, Al toxicity might not be restricted to the root tip cells, but can also act on the basal root cells. This result is consistent with a previous report that $\mathrm{Al}$ could affect the expression of some genes in both the root tip and basal root region of rice [43]. GO and KEGG enrichment analysis revealed that genes categorized into "Response to stimulus", "Antioxidant activity" and "Lipid metabolism" were preferentially induced in expression by $\mathrm{Al}$ stress (Tables 2 and 3), which supported previous observations that $\mathrm{Al}$ can induce the peroxidation of lipids and the production of reactive oxygen species (ROS), and that plant roots are able to increase the expression of antioxidant genes such as glutathione S-transferase (GST) genes to cope with $\mathrm{Al}$ toxicity $[34,44,45]$. Additionally, the expression of genes categorized as "Extracellular" or putatively involved in "Carbohydrate metabolism" were also increased in the root tips in response to $\mathrm{Al}$, which was consistent with the concept that the root cell wall is the primary target site of Al toxicity $[2,44]$.

Both common and tartary Buckwheat are able to secrete oxalate to chelate and detoxify $\mathrm{Al}$ in the rhizosphere $[13,14,30]$, although the genes responsible for the release of oxalate from the roots have not been identified. There are two temporal patterns adopted by plants for Al-activated organic acid release [27]. In Pattern I, exudation of organic acids is rapidly activated by $\mathrm{Al}$ exposure and there is no discernible delay observed between the addition of $\mathrm{Al}$ and the onset of organic acid anion release, whereas in Pattern II the secretion of organic acids is delayed for several hours after exposure to Al. The secretion of oxalate in buckwheat is rapid and at
A

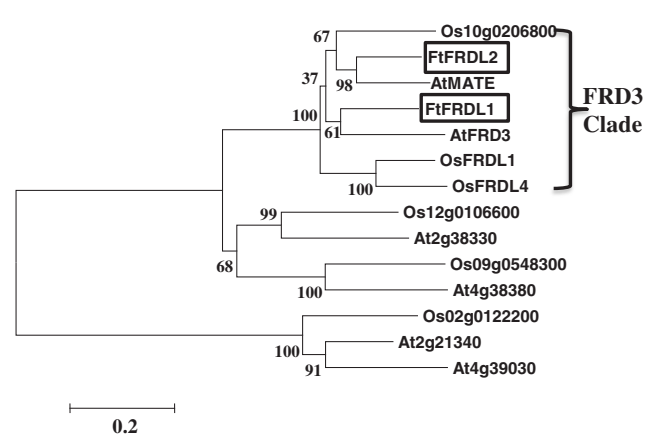

B

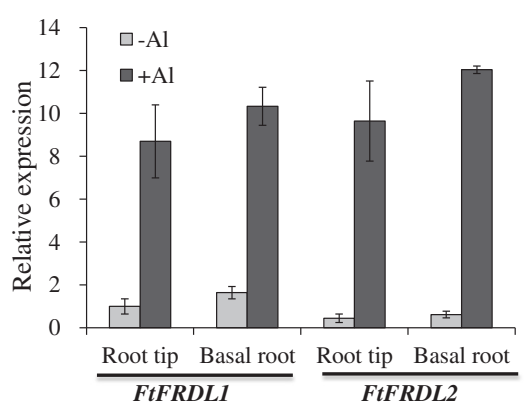

Figure 6 Phylogenetic and expression analysis of FRD3 homologs in buckwheat. (A) Phylogenetic tree of buckwheat FRD3 homologs (boxed FtFRDL1 and FtFRDL2) and other MATE homologs from Arabidopsis and rice. (B) Effect of Al stress on the expression of FtFRDL1 and FtFRDL2 in different root regions. The data were normalized to FtFRDL1 expression in the root tips without Al treatment. Data shown are means \pm SD ( $n=3$ ). 


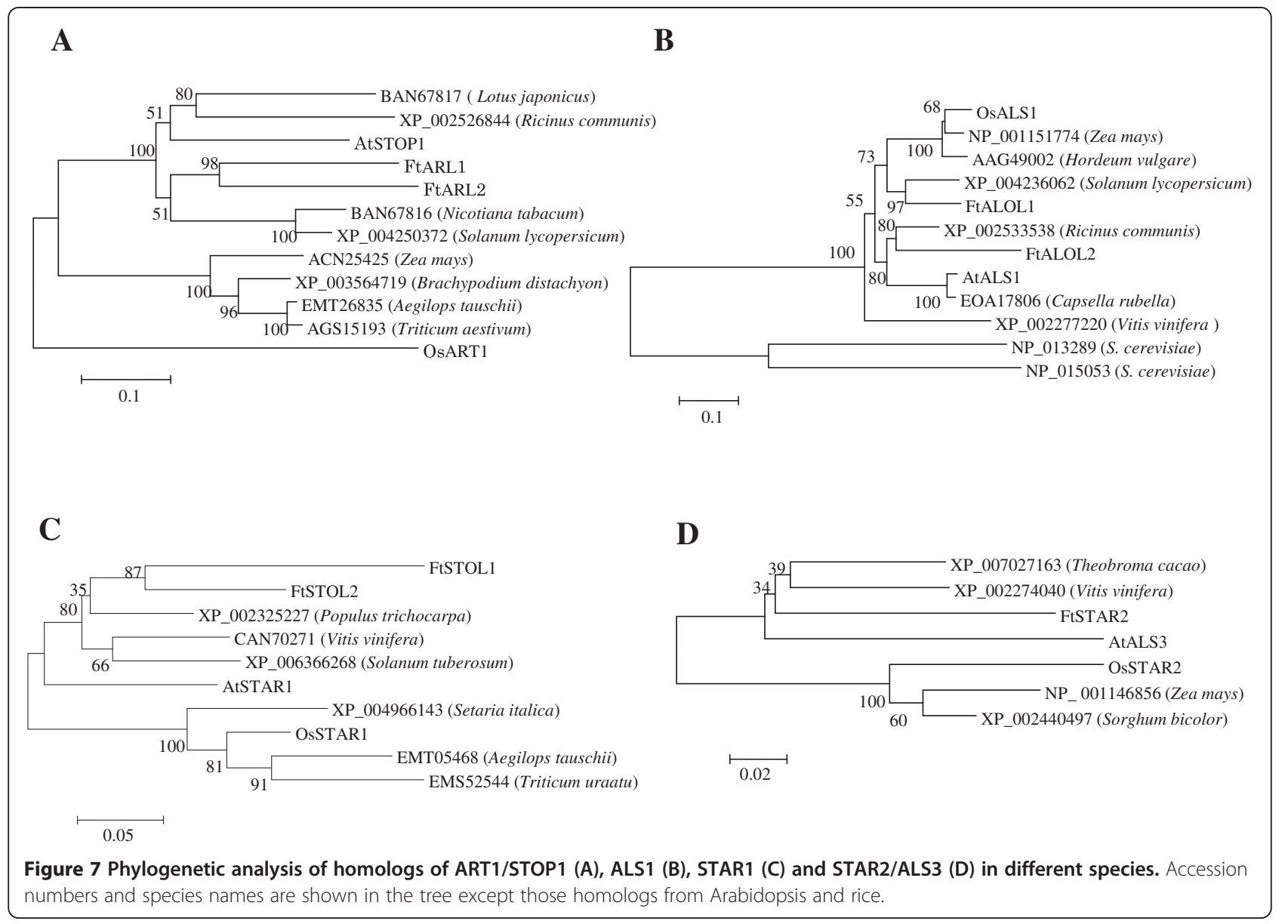

a constant level after the exposure to $\mathrm{Al}[13,14]$, consistent with a Pattern I response. Recent reports on wheat ALMT1 and barley HvAACT1 indicate that the expression of genes encoding transporters for the secretion of organic acid in Pattern I is constitutive and not responsive to $\mathrm{Al}$ stress $[17,18]$. Therefore, it will be difficult to use RNA-seq analysis to identify the genes responsible for the exudation of oxalate in buckwheat since their expression might not be affected by $\mathrm{Al}$ stress. An alternative approach could be to screen mutants defective in oxalate secretion, followed by cloning of the responsive genes through map-based cloning techniques, to isolate genes encoding oxalate transporters.

Interestingly, we found that the expression of two homologs of FRD3 was highly induced by the $\mathrm{Al}$ treatment (Figure 6B). The MATE genes in the FRD3 subgroup have been demonstrated to be involved in the translocation of iron through the release of citrate to the xylem or in $\mathrm{Al}$ tolerance through citrate release to the rhizosphere in Arabidopsis [41,42]. Although buckwheat secretes oxalate instead of citrate to the rhizosphere for the detoxification of $\mathrm{Al}$, it is possible that the plant may release citrate to the xylem for the translocation of $\mathrm{Al}$ because the $\mathrm{Al}$-citrate complex is the predominant form of $\mathrm{Al}$ in the xylem [29], which could be mediated by the FRD3-like transporters in buckwheat. Similarly, release of citrate into the xylem is required for iron translocation in both dicot and monocot species $[41,46]$. The requirement for citrate in the xylem translocation of both iron and $\mathrm{Al}$ in buckwheat would need to be coordinated closely. Because buckwheat hyperaccumulates $\mathrm{Al}$ in the shoots, the amount of citrate required for $\mathrm{Al}$ translocation in the xylem could be substantial. In the presence of $\mathrm{Al}$, the amount of citrate release to the xylem would have to be increased, triggering the induction of genes involved in citrate release. The increased expression of the two FRD3 homologous genes under $\mathrm{Al}$ treatment supports our speculation. In the future, it will be critical to determine whether the two genes are involved in the translocation of $\mathrm{Al}$ and/or iron in buckwheat and to examine how $\mathrm{Al}$ activates the expression of the two genes.

The requirement of ART1/STOP1, ALS1, STAR1 and STAR2/ALS3 for Al tolerance appears to be conserved and ubiquitous in monocot and dicot species, and they do not have close homologs in the rice and Arabidopsis 
A

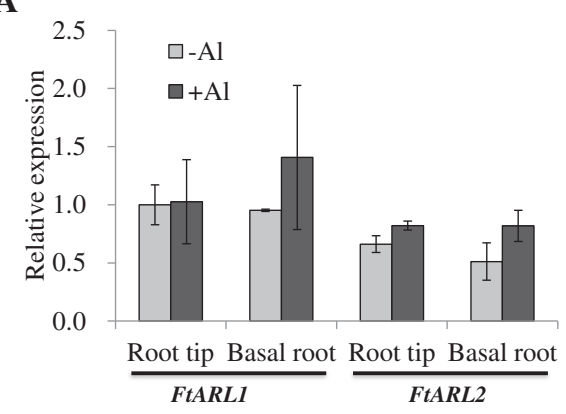

$\mathbf{C}$

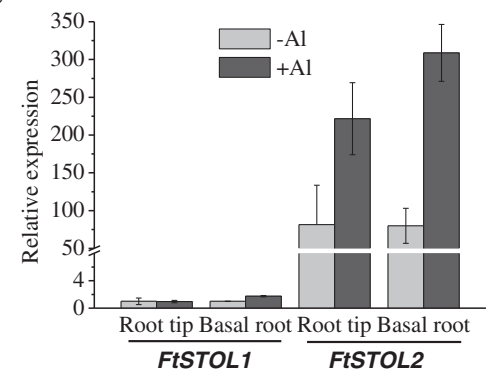

B

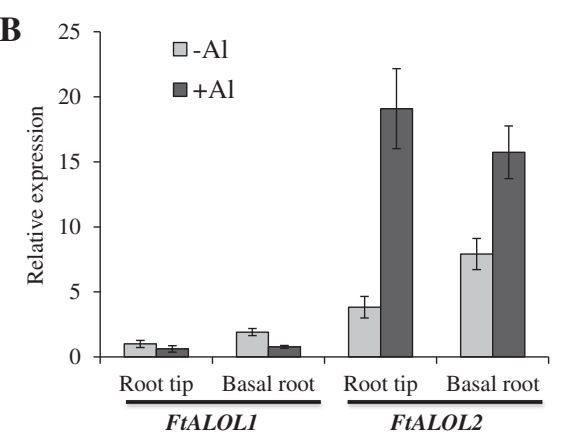

D

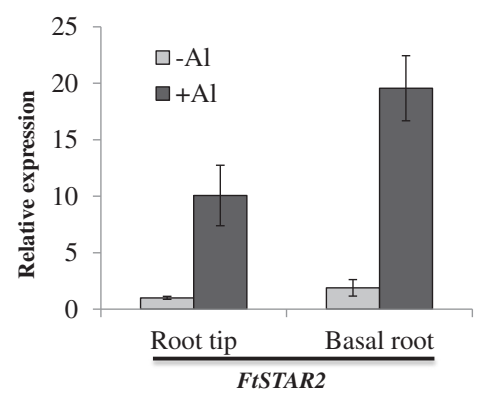

Figure 8 Expression analysis of Al-tolerance gene homologs in different root regions under different Al conditions. (A) ART1 homologs, FtARL1 and FtARL2. (B) ALS1 homologs, FtALOL1 and FtALOL2. (C) STAR1 homologs, FtSTOL1 and FtSTOL2. (D) FtSTAR2. The data were normalized to the expression of gene homolog1 in the root tips without Al treatment. Data shown are means \pm SD $(n=3)$.

genomes [20-26]. By contrast, we found that three of the four genes had been duplicated in buckwheat (Figure 7). The two homologs of $A R T 1$, a putative zinc-finger transcription factor, were expressed at a similar level and largely unaffected by the $\mathrm{Al}$ stress (Figure 8A), similar to the expression pattern of $A R T 1$ and STOP1. It remains to be demonstrated whether the two homologs are redundant or have different tissue-specific expression patterns. It will be also interesting to investigate whether the two ART1 homologs are required for Al translocation and accumulation in the shoots of buckwheat. In contrast to the ART1 homologs, the two homologs of STAR1, a putative $\mathrm{ABC}$ transporter, displayed an unequal expression pattern, with FtSTOL2 accumulating to a higher level than FtSTOL1 in the roots (Figure 8C). Furthermore, the expression of FtSTOL2 was highly induced by the $\mathrm{Al}$ treatment, whereas that of FtSTOL1 was unaffected. These results suggest that FtSTOL2 is the major gene required for $\mathrm{Al}$ tolerance in the roots of buckwheat. Although FtSTOL2 had greater sequence similarity to Arabidopsis AtSTAR1 than to rice STAR1 (Figure 7C), the expression pattern of FtSTOL2 was similar to rice STAR1. Arabidopsis AtSTAR1 is mainly expressed in the root tip region and is not responsive to Al stress [23], whereas both buckwheat FtSTOL2 and rice STAR1 were equally expressed in both the root tip and basal root region and their expression was highly induced by $\mathrm{Al}$ [22]. Unlike STAR1 homologs, there was only one homolog of STAR2 in buckwheat. The expression of FtSTAR2 was also greatly increased after exposure to $\mathrm{Al}$ stress (Figure 8D), which reinforced the view that Alinduced expression of STAR2 is a conserved mechanism in plants since previous reports also showed that rice STAR2 and Arabidopsis ALS3 were increased in expression after exposure to $\mathrm{Al}[22,24]$.

Whereas the duplication of ART1/STOP1 and STAR1 appears to occur after the divergence of dicots and monocots, ALS1 duplication may have occurred before the split of monocots and dicots (Figure 7). In fact, duplication of ALS1 appears to be an ancient event because the yeast Saccharomyces cerevisiae has two copies of ALS1 in its genome (Figure 7B). While many plants appear to have lost one copy of ALS1, tartary buckwheat retains both copies. We also found that tea (Camellia sinensis) has two copies of ALS1 (Unpublished data). As both buckwheat and tea are $\mathrm{Al}$ hyperaccumulators and highly tolerant to $\mathrm{Al}$ stress, these results suggest that retaining two ALS1 copies might be a common feature for Al hyperaccumulators with both homologs playing important roles in the tolerance and/or distribution of Al. In addition, although phylogenetic analysis showed that FtALOL2 was closer to Arabidopsis AtALS1 (Figure 7B), the expression pattern of FtALOL2 was more like that of rice OsALS1 (Figure 8B). AtALS1 was preferentially 
expressed in the root tip region and its expression was not affected by the $\mathrm{Al}$ treatment [25], whereas both FtALOL2 and OsALS1 had greater expression in the basal roots than in the root tips, and their expression was induced by $\mathrm{Al}$ in both root regions [26]. Conversely, FtALOL1 expression was not induced by $\mathrm{Al}$ stress even though FtALOL1 had greater sequence similarity to the monocot OsALS1 (which is upregulated by $\mathrm{Al}$ stress) than to the dicot AtALS1 (Figures 7B and 8B). In the future, it will be essential to determine the in vivo function of the two ALS1 homologs in buckwheat and to examine whether they have redundant functions in $\mathrm{Al}$ tolerance and/or accumulation in roots and shoots of buckwheat.

Compared with the Al-sensitive species Arabidopsis, the Al-tolerance species rice is able to express high levels of the conserved $\mathrm{Al}$-tolerance genes in the presence of $\mathrm{Al}$ to overcome Al stress. Similar to rice, tartary buckwheat also showed high expression of the Al-tolerance gene homologs under $\mathrm{Al}$ stress, although the $\mathrm{Al}$-tolerance species buckwheat is evolutionarily closer to Arabidopsis than rice (Figures 7 and 8). These suggest that buckwheat has evolved high expression of Al-tolerance genes to detoxify Al. In addition, buckwheat has experienced gene duplication of ART1/STOP1, STAR1 and ALS1. Since buckwheat can accumulate high levels of $\mathrm{Al}$ in addition to having high tolerance to $\mathrm{Al}$, gene duplication might be important for buckwheat to coordinate the $\mathrm{Al}$ tolerance and $\mathrm{Al}$ accumulation in roots and shoots. In this regard, it is interesting to note that zinc/cadmium hyperaccumulation in Arabidopsis halleri also involves duplication of key genes responsible for metal translocation and detoxification [47]. Further functional analysis by creating knock-down or knock-out mutants will be required to reveal the role of each homologous gene in $\mathrm{Al}$ detoxification and accumulation in buckwheat.

\section{Conclusions}

Through genome-wide mRNA sequencing analysis, we constructed about 40,000 high-quality transcripts in tartary buckwheat, which provide a sequence basis for further investigation into the molecular mechanisms of $\mathrm{Al}$ tolerance and accumulation in buckwheat. Our RNA-seq analysis reveals that the root tip and the basal root region of tartary buckwheat may possess both common and different mechanisms of $\mathrm{Al}$ responsiveness and that organic acid metabolism is not the rate-limiting step for organic acid secretion induced by $\mathrm{Al}$ in buckwheat. We propose that xylem loading of $\mathrm{Al}$ may be a rate-limiting step for the translocation of $\mathrm{Al}$ from roots to shoots in buckwheat and that two putative citrate transporters, FtFRDL1 and FtFRDL2, may be required for the translocation of $\mathrm{Al}$ via the release of citrate into the xylem for complexation with Al. We also propose that buckwheat has experienced duplication and subfunctionlization of key genes to coordinate the $\mathrm{Al}$ tolerance and $\mathrm{Al}$ accumulation.

\section{Methods}

Plant materials and growth conditions

Wild-type buckwheat used for transcriptome analysis was Fagopyrum tataricum (cv. Xiqiao2). The Xiqiao2 variety is widely cultivated in Liangshan prefecture of Sichuan province in China and we collected its seeds at a food market in that area. Seeds were soaked in deionized water for $6 \mathrm{~h}$ in the dark at room temperature and then transferred to nets floating on a $0.5 \mathrm{mM} \mathrm{CaCl} 2$ solution in a 3-liter plastic container. The solution was renewed every day. Plants were grown in a growth chamber at $23^{\circ} \mathrm{C}$ in the dark. Three days later, the seedlings were pretreated with a $0.5 \mathrm{mM} \mathrm{CaCl}$ solution at $\mathrm{pH} 4.5$ for $24 \mathrm{~h}$ before being exposed to a $0.5 \mathrm{mM} \mathrm{CaCl}_{2}$ solution containing 0 or $50 \mu \mathrm{M} \mathrm{AlCl}_{3}$ at $\mathrm{pH} 4.5$ for $6 \mathrm{~h}$. Root tips $(0-2 \mathrm{~cm})$ and basal roots $(2-4 \mathrm{~cm})$ with three biological replicates were sampled in both $-\mathrm{Al}$ and $+\mathrm{Al}$ conditions for RNA-seq. For each sample, 40-50 root segments were collected and frozen in liquid nitrogen within $5 \mathrm{~min}$ for RNA isolation. Due to the cost consideration, RNA-seq was performed on two replicates of root tips and one replicate of basal roots in both $-\mathrm{Al}$ and $+\mathrm{Al}$ treatments. For real-time RT-PCR analysis, all the three replicates were used to quantify the gene expression. It has been shown that the reliability of differential expression in RNA-seq is dependent on the sequencing depth [48]. To ensure reliability, our samples were sequenced to around 250 fold coverage of each contig on average (Additional file 2: Table S2). Furthermore, the RNA-seq data were verified by quantitative RT-PCR (Figure 4).

\section{Determination of Al accumulation}

For determination of $\mathrm{Al}$ concentrations in roots and shoots, two-week-old seedlings of tartary buckwheat (cv. Xiqiao2) and common buckwheat (cv. Jiangxi) were exposed to a $0.5 \mathrm{mM} \mathrm{CaCl}_{2}$ solution containing $0,10,20$ or $50 \mu \mathrm{M} \mathrm{AlCl}_{3}$ for $24 \mathrm{~h}$ and then to one-fifth strength Hoagland's solution for another $24 \mathrm{~h}$. After intermittent $\mathrm{Al}$ treatment for $8 \mathrm{~d}$, roots and shoots were sampled for the determination of $\mathrm{Al}$ concentrations. The samples were dried at $60^{\circ} \mathrm{C}$ in an oven for a week and digested with $\mathrm{HNO}_{3}$. The $\mathrm{Al}$ concentration was measured by Inductively Coupled Plasma Mass Spectrometry (Nexion 300X ICP-MS, Perkin Elmer, USA).

\section{RNA isolation, library construction and Illumina deep sequencing}

Total RNA was extracted using General Plant RNA Extraction Kit (BioTeke, China). The extracted RNA was digested with DNase I (TAKARA) to remove contaminated DNA. mRNAs were purified from the total RNA 
using Dynabeads Oligo $(\mathrm{dT})_{25}$ (Life Technologies). The derived mRNAs were fragmented and reverse transcribed into first-strand cDNAs with random hexamer and then the second-strand cDNAs were synthesized by using a NEBNext UltraTM RNA Library Prep Kit for Illumina (NEB). The double-stranded cDNAs were purified and ligated to adaptors for Illumina paired-end sequencing. The cDNA library was sequenced using the Illumina HiSeq2500 system by Shanghai Hanyu Biotech lab (Shanghai, China).

\section{Transcriptome assembly and annotation}

Raw reads were filtered using the FastaX package to remove adaptor sequences and low-quality reads (base quality $<20$, read length $<40 \mathrm{bp}$ ). The obtained clean reads of all six samples were assembled using the Trinity program [32] with the following parameters: $\mathrm{k}$-mer $=25$; minimum $\mathrm{k}$-mer coverage $=2$; maximum length expected between fragment pairs $=500$; minimum overlap of reads with growing transcript $\mathrm{PE}=75$; maximum number of reads to anchor within a single graph $=200,000$. Putative coding sequences of the assembled transcripts were predicted by "get orf" in the EMBOSS package. To annotate the assembled transcripts, BLASTp searches (e-value $<0.00001$ ) were performed among all-predicted coding sequences and protein databases including NCBI, Swiss-Prot, KEGG and COG. Functional annotation using gene ontology terms (GO; http://www.geneontology.org) was analyzed using the BLASTp algorithm against the Swiss Prot database by the GoPipe program of gene2go software at $\mathrm{ftp}: / / 203.110 .175 .109$. COG/KOG and KEGG pathways annotation was carried out using Blastall software against the Cluster of Orthologous Groups database and Kyoto Encyclopedia of Genes and Genomes database, respectively.

\section{Differential expression analysis and GO and KEGG enrichment analysis}

The cleaned reads of each sample were mapped back to assembled contigs by bowtie 2 with the following parameters: maximum mismatches in seed alignment $=1$; length of seed substrings $=22$. The assembled contigs with more than 10 reads mapped were subjected to differential expression analysis. For the expression analysis, the number of clean reads for each contig was calculated and then normalized to Reads Per Kb per Million reads (RPKM). The expression difference of each contig between different treatments was calculated based on the MARS model (MA-plot-based method with Random Sampling model) using the DEGseq package. FDR (false discovery rate) value less than 0.001 and $\mid \log _{2}$ (fold change) $\mid \geq 1$ were used as the threshold to judge the significance of gene expression difference.

Differentially expressed genes were extracted for GO functional enrichment analysis and KEGG pathway enrichment analysis. The enrichment analysis was tested using a hypergeometric test at a significance cutoff of $\sim 0.1 \%$ false discovery rate (FDR).

\section{Real-time RT-PCR}

One microgram of total RNA was used to synthesize the first-strand cDNAs by using HiScript ${ }^{\circ} 1$ st Strand cDNA Synthesis Kit (Vazyme). One twentieth of the cDNA products and the $\mathrm{SYBR}^{\circ}$ Green Master Mix kit (Vazyme) were used for real-time RT-PCR analysis. The SAND gene was used as the internal control, which has been shown to be one of most stable reference genes in buckwheat [49]. Primers for real-time RT-PCR analysis are listed in Additional file 6: Table S5. Data were collected in accordance with the CFX96 Real-Time PCR Detection System (Bio-Rad).

\section{Phylogenetic analysis}

Phylogenetic analysis was carried out by using the MEGA4.0 program (http://www.megasoftware.net). The neighbor-joining method was used to construct the phylogenetic tree with 1000 bootstrap trials by MEGA4.0 [50].

\section{Availability of supporting data}

Illumina high throughput mRNA sequencing data of Fagopyrum tataricum (cv. Xiqiao2) were deposited in the NCBI SRA database under following accession numbers: SRR1460477 and SRR1552100 (two replicates, root tip region, -Al condition), SRR1552203 and SRR1552215 (two replicates, root tip region, $+\mathrm{Al}$ condition), SRR1552101 (basal root region, -Al condition), and SRR1552217 (basal root region, $+\mathrm{Al}$ condition). The phylogenetic trees were deposited in treebase (http://treebase.org) under follo wing URL: http://purl.org/phylo/treebase/phylows/study/ TB2:S16751? $x$-access-code $=3031 f 75 c f 503 d 89 e 82 b 52 c 6 b a 8$ $769 \mathrm{~d} 97 \&$ format=html. The data sets supporting the results of this article are included within the article and its additional files.

\section{Additional files}

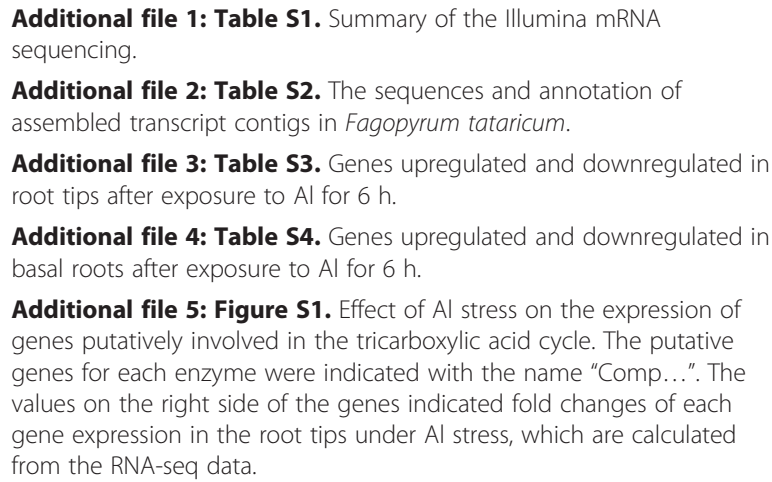

Additional file 2: Table S2. The sequences and annotation of assembled transcript contigs in Fagopyrum tataricum.

Additional file 3: Table S3. Genes upregulated and downregulated in root tips after exposure to Al for $6 \mathrm{~h}$.

Additional file 4: Table S4. Genes upregulated and downregulated in basal roots after exposure to Al for $6 \mathrm{~h}$.

Additional file 5: Figure S1. Effect of Al stress on the expression of genes putatively involved in the tricarboxylic acid cycle. The putative genes for each enzyme were indicated with the name "Comp...". The values on the right side of the genes indicated fold changes of each gene expression in the root tips under Al stress, which are calculated from the RNA-seq data.

Additional file 6: Table S5. Primers used for quantitative RT-PCR analysis. 


\section{Abbreviations}

Al: Aluminum; GO: Gene Ontology; KEGG: Kyoto Encyclopedia of Genes and Genomes; MATE: Multidrug and toxic compound extrusion; ORF: Open reading frame; RACE: Rapid-amplification of cDNA ends; RNA-seq: Highthroughput RNA sequencing; ROS: Reactive oxygen species.

\section{Competing interests}

The authors declare that they have no competing interests.

\section{Authors' contributions}

CFH designed and supervised the research. HZ, HW, JZ and CFH performed RNA-seq data analysis; $\mathrm{HZ}, \mathrm{HW}$ and $\mathrm{YZ}$ performed $\mathrm{Al}$ accumulation and expression analysis. CFH and FJZ wrote the manuscript. All authors read and approved the final manuscript.

\section{Acknowledgements}

We thank Dr. Jacquie De-Silva for commenting on the manuscript. This work was supported by the National Natural Science Foundation of China (Grant No. 31272224 to C.-F. H.), the Fundamental Research Funds for the Central Universities (Grant No. KYRC201207 and KYTZ201404), the Program for Changjiang Scholars and Innovative Research Team in University (Grant No. IRT1256) and the Priority Academic Program Development of Jiangsu Higher Education Institutions (PAPD).

\section{Author details}

${ }^{1}$ State Key Laboratory of Crop Genetics and Germplasm Enhancement, College of Resources and Environmental Science, Nanjing Agricultural University, Nanjing 210095, China. ${ }^{2}$ State Key Laboratory Breeding Base for Zhejiang Sustainable Pest and Disease Control, Institute of Crop and Nuclear Technology Utilization, Zhejiang Academy of Agricultural Sciences, Hangzhou 310021, China.

Received: 23 August 2014 Accepted: 22 December 2014 Published online: 21 January 2015

\section{References}

1. Vonuexkull HR, Mutert E. Global extent, development and economic-impact of acid soils. Plant Soil. 1995;171(1):1-15.

2. Kochian LV. Cellular mechanisms of aluminum toxicity and resistance in plants. Annu Rev Plant Phys. 1995;46:237-60.

3. Ma JF. Role of organic acids in detoxification of aluminum in higher plants. Plant Cell Physiol. 2000;41(4):383-90.

4. Ryan $P$, Delhaize $E$, Jones D. Function and mechanism of organic anion exudation from plant roots. Annu Rev Plant Phys. 2001;52:527-60.

5. Delhaize E, Ryan PR, Randall PJ. Aluminum tolerance in wheat (Triticum aestivum L.) 2. Aluminum-stimulated excretion of malic-acid from root apices. Plant Physiol. 1993;103(3):695-702.

6. Ligaba A, Katsuhara M, Ryan PR, Shibasaka M, Matsumoto H. The BnALMT1 and BnALMT2 genes from rape encode aluminum-activated malate transporters that enhance the aluminum resistance of plant cells. Plant Physiol. 2006;142(3):1294-303.

7. Hoekenga OA, Vision TJ, Shaff JE, Monforte AJ, Lee GP, Howell SH, et al. Identification and characterization of aluminum tolerance loci in Arabidopsis (Landsberg erecta $\times$ Columbia) by quantitative trait locus mapping, A physiologically simple but genetically complex trait. Plant Physiol. 2003;132(2):936-48.

8. Miyasaka SC, Buta JG, Howell RK, Foy CD. Mechanism of aluminum tolerance in snapbeans : root exudation of citric acid. Plant Physiol. 1991;96 (3):737-43.

9. Pellet DM, Grunes DL, Kochian LV. Organic-acid exudation as an aluminumtolerance mechanism in maize (Zea mays L.). Planta. 1995;196(4):788-95.

10. Yang ZM, Sivaguru $M$, Horst WJ, Matsumoto $H$. Aluminium tolerance is achieved by exudation of citric acid from roots of soybean (Glycine max). Physiol Plantarum. 2000;110(1):72-7.

11. Liu MY, Chen WW, Xu JM, Fan W, Yang JL, Zheng SJ. The role of VuMATE1 expression in aluminium-inducible citrate secretion in rice bean (Vigna umbellata) roots. J Exp Bot. 2013:64(7):1795-804.

12. Yang JL, Zhang L, Li YY, You JF, Wu P, Zheng SJ. Citrate transporters play a critical role in aluminium-stimulated citrate efflux in rice bean (Vigna umbellata) roots. Ann Bot-London. 2006;97(4):579-84.
13. Ma JF, Zheng SJ, Matsumoto H, Hiradate S. Detoxifying aluminium with buckwheat. Nature. 1997:390(6660):569-70.

14. Zheng SJ, Ma JF, Matsumoto H. High aluminum resistance in buckwheat. I. Al-induced specific secretion of oxalic acid from root tips. Plant Physiol. 1998;117(3):745-51.

15. Yang JL, Zheng SJ, He YF, Matsumoto $H$. Aluminium resistance requires resistance to acid stress: a case study with spinach that exudes oxalate rapidly when exposed to Al stress. J Exp Bot. 2005;56(414):1197-203.

16. Yang JL, Zhu XF, Peng YX, Zheng C, Ming F, Zheng SJ. Aluminum regulates oxalate secretion and plasma membrane $\mathrm{H}+-$ ATPase activity independently in tomato roots. Planta. 2011;234(2):281-91.

17. Sasaki T, Yamamoto Y, Ezaki B, Katsuhara M, Ahn SJ, Ryan PR, et al. A wheat gene encoding an aluminum-activated malate transporter. Plant J. 2004:37(5):645-53.

18. Furukawa J, Yamaji N, Wang H, Mitani N, Murata Y, Sato K, et al. An aluminum-activated citrate transporter in barley. Plant Cell Physiol. 2007:48(8):1081-91.

19. Magalhaes JV, Liu J, Guimaraes CT, Lana UGP, Alves VMC, Wang YH, et al. A gene in the multidrug and toxic compound extrusion (MATE) family confers aluminum tolerance in sorghum. Nat Genet. 2007;39(9):1156-61.

20. Iuchi S, Koyama H, luchi A, Kobayashi Y, Kitabayashi S, Ikka T, et al. Zinc finger protein STOP1 is critical for proton tolerance in Arabidopsis and coregulates a key gene in aluminum tolerance. Proc Natl Acad Sci U S A. 2007;104(23):9900-5

21. Yamaji N, Huang CF, Nagao S, Yano M, Sato Y, Nagamura $Y$, et al. A zinc finger transcription factor ART1 regulates multiple genes implicated in aluminum tolerance in rice. Plant Cell. 2009;21(10):3339-49.

22. Huang CF, Yamaji N, Mitani N, Yano M, Nagamura Y, Ma JF. A bacterial-type $A B C$ transporter is involved in aluminum tolerance in rice. Plant Cell. 2009;21(2):655-67.

23. Huang CF, Yamaji N, Ma JF. Knockout of a bacterial-type ATP-binding cassette transporter gene, AtSTAR1, results in increased aluminum sensitivity in Arabidopsis. Plant Physiol. 2010;153(4):1669-77.

24. Larsen PB, Geisler MJB, Jones CA, Williams KM, Cancel JD. ALS3 encodes a phloem-localized $A B C$ transporter-like protein that is required for aluminum tolerance in Arabidopsis. Plant J. 2005:41(3):353-63.

25. Larsen PB, Cancel J, Rounds M, Ochoa V. Arabidopsis ALS1 encodes a root tip and stele localized half type $A B C$ transporter required for root growth in an aluminum toxic environment. Planta. 2007;225(6):1447-58.

26. Huang CF, Yamaji N, Chen ZC, Ma JF. A tonoplast-localized half-size ABC transporter is required for internal detoxification of aluminum in rice. Plant J. 2012;69(5):857-67.

27. Ma JF, Ryan PR, Delhaize E. Aluminium tolerance in plants and the complexing role of organic acids. Trends Plant Sci. 2001;6(6):273-8.

28. Ma JF, Hiradate S, Matsumoto H. High aluminum resistance in buckwheat - II. Oxalic acid detoxifies aluminum internally. Plant Physiol. 1998;117(3):753-9.

29. Ma JF, Hiradate S. Form of aluminium for uptake and translocation in buckwheat (Fagopyrum esculentum Moench). Planta. 2000;211(3):355-60.

30. Wang H, Chen RF, Iwashita T, Shen RF, Ma JF. Physiological characterization of aluminum tolerance and accumulation in tartary and wild buckwheat. New Phytol. 2014. doi:10.111/nph.13011

31. Yang Y, Smith SA. Optimizing de novo assembly of short-read RNA-seq data for phylogenomics. BMC Genomics. 2013;14:328.

32. Grabherr MG, Haas BJ, Yassour M, Levin JZ, Thompson DA, Amit I, et al. Full-length transcriptome assembly from RNA-Seq data without a reference genome. Nat Biotechnol. 2011;29(7):644-52.

33. Logacheva MD, Kasianov AS, Vinogradov DV, Samigullin TH, Gelfand MS, Makeev $V J$, et al. De novo sequencing and characterization of floral transcriptome in two species of buckwheat (Fagopyrum). BMC Genomics. 2011;12:30.

34. Yamamoto $Y$, Kobayashi $Y$, Matsumoto H. Lipid peroxidation is an early symptom triggered by aluminum, but not the primary cause of elongation inhibition in pea roots. Plant Physiol. 2001;125(1):199-208.

35. Kinraide TB. Ion fluxes considered in terms of membrane-surface electrical potentials. Aust J Plant Physiol. 2001;28(7):605-16.

36. Klug B, Horst WJ. Oxalate exudation into the root-tip water free space confers protection from aluminum toxicity and allows aluminum accumulation in the symplast in buckwheat (Fagopyrum esculentum). New Phytol. 2010;187(2):380-91.

37. Zheng SJ, Yang JL, He YF, Yu XH, Zhang L, You JF, et al. Immobilization of aluminum with phosphorus in roots is associated with high aluminum resistance in buckwheat. Plant Physiol. 2005;138(1):297-303. 
38. Hayes JE, Ma JF. Al-induced efflux of organic acid anions is poorly associated with internal organic acid metabolism in triticale roots. J Exp Bot. 2003;54(388):1753-9.

39. Ryan PR, Delhaize E, Randall PJ. Characterization of Al-Stimulated efflux of malate from the apices of Al-tolerant wheat roots. Planta. 1995;196(1):103-10.

40. Maron LG, Kirst M, Mao C, Milner MJ, Menossi M, Kochian LV. Transcriptional profiling of aluminum toxicity and tolerance responses in maize roots. New Phytol. 2008;179(1):116-28.

41. Rogers EE, Guerinot ML. FRD3, a member of the multidrug and toxin efflux family, controls iron deficiency responses in Arabidopsis. Plant Cell. 2002;14(8):1787-99.

42. Liu JP, Magalhaes JV, Shaff J, Kochian LV. Aluminum-activated citrate and malate transporters from the MATE and ALMT families function independently to confer Arabidopsis aluminum tolerance. Plant J. 2009;57(3):389-99.

43. Tsutsui T, Yamaji N, Huang CF, Motoyama R, Nagamura Y, Ma JF. Comparative genome-wide transcriptional analysis of Al-responsive genes reveals novel Al tolerance mechanisms in rice. PLoS One. 2012;7(10):e48197.

44. Ma JF. Syndrome of aluminum toxicity and diversity of aluminum resistance in higher plants. Int Rev Cytol. 2007;264:225-52.

45. Ezaki B, Suzuki M, Motoda $H$, Kawamura M, Nakashima S, Matsumoto $H$. Mechanism of gene expression of Arabidopsis glutathione S-transferase, AtGST1, and AtGST11 in response to aluminum stress. Plant Physiol. 2004;134(4):1672-82.

46. Yokosho K, Yamaji N, Ueno D, Mitani N, Ma JF. OsFRDL1 is a citrate transporter required for efficient translocation of iron in rice. Plant Physiol. 2009:149(1):297-305.

47. Hanikenne M, Talke IN, Haydon MJ, Lanz C, Nolte A, Motte P, et al. Evolution of metal hyperaccumulation required cis-regulatory changes and triplication of HMA4. Nature. 2008;453(7193):391-5.

48. Tarazona S, Garcia-Alcalde F, Dopazo J, Ferrer A, Conesa A. Differential expression in RNA-seq: a matter of depth. Genome Res. 2011;21(12):2213-23.

49. Demidenko NV, Logacheva MD, Penin AA. Selection and validation of reference genes for quantitative real-time PCR in buckwheat (Fagopyrum esculentum) based on transcriptome sequence data. PLoS One. 2011:6(5):e19434.

50. Tamura K, Peterson D, Peterson N, Stecher G, Nei M, Kumar S. MEGA5: molecular evolutionary genetics analysis using maximum likelihood, evolutionary distance, and maximum parsimony methods. Mol Biol Evol. 2011;28(10):2731-9.

\section{Submit your next manuscript to BioMed Central and take full advantage of:}

- Convenient online submission

- Thorough peer review

- No space constraints or color figure charges

- Immediate publication on acceptance

- Inclusion in PubMed, CAS, Scopus and Google Scholar

- Research which is freely available for redistribution 